\title{
Anaplasma platys-Like Infection in Goats, Beijing, China
}

\author{
Wei Wei, ${ }^{1,2}$ Jie Li, ${ }^{2}$ Ya-Wei Wang, ${ }^{2}$ Bao-Gui Jiang, ${ }^{2}$ Hong-Bo Liu, ${ }^{2,3}$ Ran Wei, ${ }^{2}$ Rui-Ruo Jiang,,4 \\ Xiao-Ming Cui, ${ }^{2}$ Lian-Feng Li, ${ }^{2,5}$ Ting-Ting Yuan, ${ }^{2,6}$ Qian Wang, ${ }^{2}$ Lin Zhao, ${ }^{2}$ Luo-Yuan Xia, ${ }^{2}$ \\ Jia-Fu Jiang, ${ }^{2}$ Ye-Feng Qiu, ${ }^{7} \mathrm{Na} \mathrm{Jia},{ }^{2}$ Wu-Chun $\mathrm{CaO}^{2},{ }^{2}$ and Yan-Ling $\mathrm{Hu}^{1}$
}

\begin{abstract}
As one of the important tick-borne zoonotic pathogens, Anaplasma has both veterinary and public health significance. Here, we performed a survey of Anaplasma infection in the goats from a farm in Beijing, China, and found $44.6 \%$ (41/92) were infected with Anaplasma capra, and 22.8\% (21/92) were infected with Anaplasma sp. This Anaplasma sp. bacterium was close to a recently emerging Anaplasma platys strain based on gltA and groEL gene phylogenetic analysis. As to further understand the characteristics of Anaplasma sp., we raised a couple of positive goats $(n=2)$ in the laboratory with tick-free settings. We observed inappetence, vomiting, high fever, and weakness of limbs in the goat's offspring $(n=3)$. In addition, the blood samples from all offspring were all positive of this Anaplasma spp. We did not see any intracellular morulae in neutrophils, monocytes, and erythrocytes, but we identified some in the platelets of the blood smears from the positive goats by light microscopy. We named it A. platys-like and suggested it may infect platelets and be transmitted vertically through the placenta of goats. These findings deserve further evaluation.
\end{abstract}

Keywords: Anaplasma platys-like, goats, platelets, vertical transmission

\section{Introduction}

$\mathbf{T}$ ICK-BORNE DISEASES (TBDs) have been an increasing threat on animal production, welfare, and human health worldwide (Torina et al. 2007, Perronne 2014). The Anaplasma spp. are tick-borne pathogens with mammalian hosts in temperate, subtropical, and tropical regions of the world (Geurden et al. 2008, Tay et al. 2014, Yang et al. 2018). Anaplasma is an intracellular gram-negative bacterium, which belongs to the Anaplasmataceae family and Rickettsiales order (Dumler et al. 2001). In recent years, the public health significance of Anaplasma as emerging pathogens has also been realized (Ismail et al. 2010, Botelho-Nevers et al. 2012, Stuen et al. 2013). Anaplasma ovis, Anaplasma centrale, Anaplasma bovis, Anaplasma platys, Anaplasma phagocytophilum, and Anaplasma marginale are recognized species contained in the genus Anaplasma (Dumler et al. 2001). Recently, Anaplasma capra (Li et al. 2015a) and Anaplasma odocoilei (Tate et al. 2013) species have been included in the Anaplasma genus. The different species have diverse blood cell tropisms (Silaghi et al. 2017).

Anaplasmosis is one of the common tick-borne zoonotic diseases in domestic animals, such as sheep and goats on pastures. Clinical characteristics of infected animals depend on animal welfare and immune status (Čobádiová et al. 2013). The clinical symptoms of infected animals include inappetence, weight loss, sudden high fever, reduced milk production, abortions, and even death (Mathieson 1991, Stuen 2007, Liu et al. 2012, Yasini et al. 2012). Furthermore, infected animals are more susceptible to be coinfected by other pathogens (Čobádiová et al. 2013). For example, A. phagocytophilum is not only an aborticide but also associated with various bacterial opportunistic infections in sheep farms in northern Spain (Garcia-Perez et al. 2003). In addition, the human NY-18 A. phagocytophilum isolate can infect sheep and transmit to the lamb with some clinical symptoms (Reppert et al. 2013). Vertical transmission of A. ovis can cause death of neonatal elk calf (Hendrix et al. 2019).

\footnotetext{
${ }^{1}$ Life Sciences Institute, Guangxi Medical University, Nanning, China.

${ }^{2}$ State Key Laboratory of Pathogen and Biosecurity, Beijing Institute of Microbiology and Epidemiology, Beijing, China.

${ }^{3}$ Center for Disease Control and Prevention of PLA, Beijing, China.

${ }^{4}$ Institute of NBC Defense, Yangfang, Beijing, China.

${ }^{5}$ School of Information and Management, Guangxi Medical University, Nanning, China.

${ }^{6}$ Shanghai Institute for Emerging and Re-emerging Infectious Diseases, Shanghai Public Health Clinical Center, Shanghai, China.

${ }^{7}$ Laboratory Animal Center, Academy of Military Medical Sciences, Beijing, China.
} 
The previous study has indicated that there was a high incidence of anaplasmosis in the goats in the suburban areas of Beijing, China (Zhang et al. 2012). In 2012-2013, we performed a survey and found $A$. capra infections among the goats from a farm in Fangshan District, another suburban region of Beijing. The goats in this farm were usually grazing on the mountains, where Haemaphysalis longicornis had a high density in the peak season (Li et al. 2015a). In this study, we continued the survey in this farm and identified A. capra and a new Anaplasma spp. To understand the characteristics of this new species, we brought a couple of positives from this farm to the laboratory for further investigation.

\section{Materials and Methods}

\section{Investigation and sample collection}

The investigation was conducted on a herd of goats $(n=92)$ from a farm in Dayuan village, Qinglonghu Town, Fangshan District, Beijing (E116 $3^{\prime}$ N39 $\left.45^{\prime} 36^{\prime \prime}\right)$, in October 2016. To assess the Anaplasma infection on the goats, 92 ethylenediaminetetraacetic acid (EDTA)-anticoagulant blood samples of the goats were collected and detected by PCR targeting $16 \mathrm{~S}$ rRNA and gltA genes. After initial screening, two adult positive goats (G1 [male], G2 [female]) were further raised in the laboratory with tick-free settings. Soon after, the couple naturally bore their offspring (G3 [female], G4 [male]), and G1 and G3 gave birth to G5 [male]). Their relationship is described in Figure 1. Those five goats were raised in the laboratory of Beijing Institute of Microbiology and Epidemiology, following the animal ethics authority regulations (IACUC-132016-004).

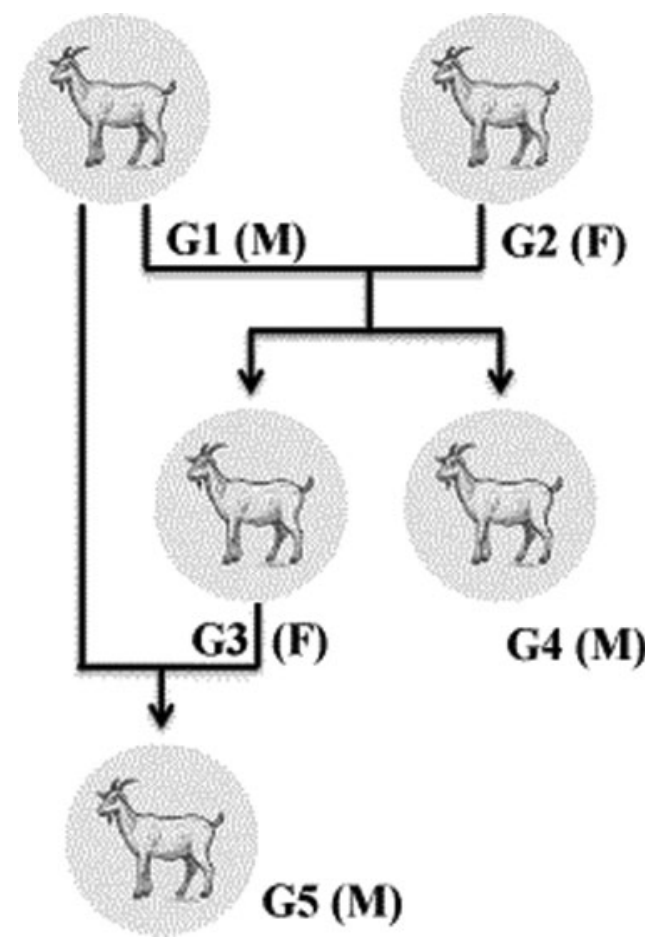

FIG. 1. G1 and G2 gave birth to G3 and G4, G1 and G3 gave birth to G5. F, female; M, male.

\section{Molecular detection and amplification of Anaplasma sp. genes}

Total DNA was extracted from all samples using the TIANamp Genomic DNA Kit (DP304; TIANGEN (Beijing) Biotech, Beijing, China) according to the kit recommended protocol. The DNA specimens were diluted into $80 \mu \mathrm{L}$ nucleic acid eluant and stored at $-20^{\circ} \mathrm{C}$. All DNA samples were tested by nested PCR using the primer pairs, targeting the $16 \mathrm{~S}$ rRNA and citrate synthase ( $g l t A)$ genes for first screening of Anaplasma. The identification of $A$. capra was followed by our previously described methods (Li et al. 2015a). To obtain the Anaplasma sp. gene sequences, DNAs of the goats' family (goat 1-goat 5, G1-G5) were amplified for the near-complete 16S rRNA, and partial gltA and groEL genes. To obtain the $16 \mathrm{~S}$ rRNA gene, the primer pair Eh-out1/Eh-3-17 was used in the first round, and the primer pairs Eh-out1/Eh-out2/Eh-out2/ Eh-3-17 were used in the second round reaction. Seminested PCR was used to amplify the gltA and groEL genes, and the primers are shown in Table 1 . Nuclease-free water was used as a negative control, and $2 \times$ DreamTaq Master Mix (Thermo Scientific, USA) including DNA polymerase was used in the PCR. To avoid cross-contamination, the experimental operations of DNA extraction, PCR preparation, DNA amplification, and agarose gel electrophoresis were in separate rooms; in addition, the template isolation and amplification were performed with the dedicated pipettes.

\section{Sequencing of PCR products and phylogenetic analysis}

All PCR products were sent to Sangon Biotech (Shanghai, China) for sequencing. The sequences obtained were checked and assembled by CLC Main Workbench 5, and were compared with the registered sequences in GenBank using the BLAST program (NCBI, Bethesda, MD) (https://blast.ncbi .nlm.nih.gov/Blast.cgi). Phylogenetic organization of Anaplasma strain revealed in this study with other referenced Anaplasma strains was constructed using the program MEGA version 5.0 software based on the DNA sequence variation of partial 16S rRNA, gltA, and groEL, respectively (Tamura et al. 2011, Li et al. 2015a).

\section{Morphological examination}

The blood smears of the goats' family (G1-G5) were prepared by air-drying and were further fixed in absolute formaldehyde and acetone 1:1 for $10 \mathrm{~min}$. Wright-Giemsa staining was then performed. The slides were rinsed with water slowly, dried, examined under the ordinary light microscope (Olympus BX41, Japan).

\section{Results}

We have tested the whole-blood samples from the 92 goats by PCR, and found that the infection rate of $A$. capra was $44.6 \%$ (41/92), and the positive rate of Anaplasma sp. was $22.8 \%$ (21/92). Anaplasma sp. infection was found in the goat couples (G1, G2) and goats G3-G5. We have obtained the sequences of near-complete $16 \mathrm{~S}$ rRNA gene $(\sim 1470 \mathrm{bp})$ and partial gltA ( $\sim 920 \mathrm{bp})$ and groEL genes ( $\sim 850 \mathrm{bp})$ for genetic identification of this Anaplasma sp. Based on the evolutionary analysis in the $16 \mathrm{~S}$ rRNA phylogenetic tree, the 
Table 1. Primer Sequences Used in This Study

\begin{tabular}{lllc}
\hline Target genes & \multicolumn{1}{c}{ Primers } & \multicolumn{1}{c}{ Oligonucleotide sequences $\left(5^{\prime}-3^{\prime}\right)$} & Target fragment $(\mathrm{bp})$ \\
\hline \multirow{2}{*}{ 16S rRNA } & Eh-out1 & TTGAGAGTTTGATCCTGGCTCAGAACG & 1470 \\
& Eh-out2 & CACCTCTACACTAGGAATTCCGCTATC & \\
EhltA & Eh-3-17 & TAAGGTGGTAATCCAGC & 920 \\
& AP1SPglF1 & ATGBTAGAAAARGCTGTTTRGMGTGT & \\
& AP1168SPglR1 & TCATACCATTGMGATRCCCATCC & \\
& APJ10F2 & AAKGCTGTTTTAGCGTGTGGTGATCTT & \\
& APJ932R2 & ATTTTCGCCCTCGGGTCGTGA & 850 \\
& Gro240F & ATTAGYAAGCCTTATGGGTC & \\
& Gro712R & CCGCGATCAAACTGCATACC & \\
& Gro481F & TGCACAGGTTGCTACTAT & \\
& Gro1059R & CDGARCTACTATCRACAC & \\
\hline
\end{tabular}

Anaplasma sp. in our study and the Anaplasma sp. in the Wen-Ping Guo study formed a cluster, and close to A. phagocytophilum, A. platys, and A. platys-like strains identified in Ankang (Fig. 2a) (Guo et al. 2019). The partial gltA gene sequence of our Anaplasma sp. formed a cluster with the
Anaplasma sp. identified in Xi' an, and this cluster was close to A. platys-like strain identified in Rhipicephalus microplus in Ankang, northwest China (Fig. 2b) (Guo et al. 2018, 2019). For the groEL gene, Anaplasma sp. found in our research was also close to the Anaplasma spp. identified in Xi'an, and it

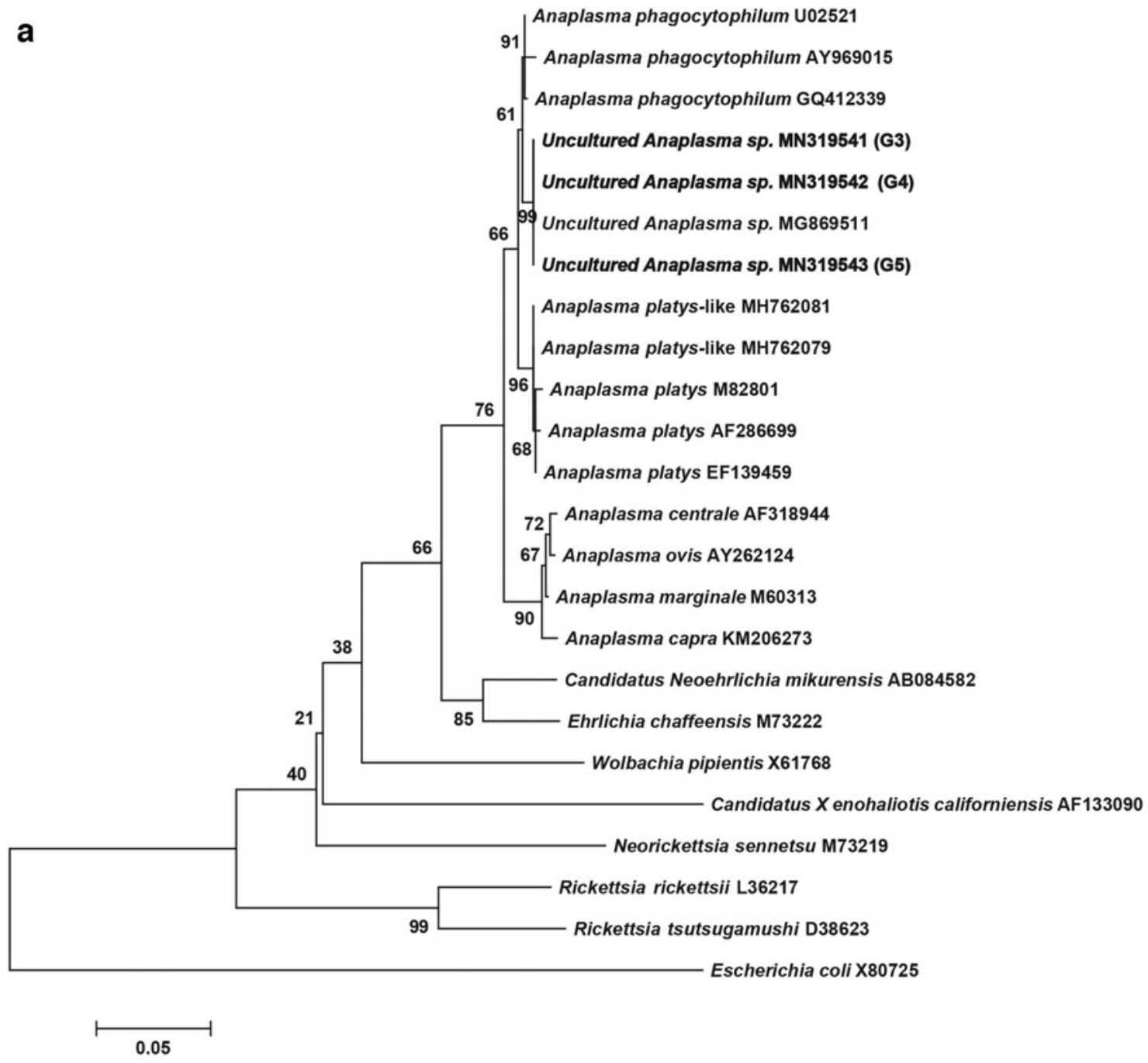

FIG. 2. Phylogenetic analyses based on nucleotide sequences of the $16 \mathrm{~S}$ rRNA (1170 bp) (a), citrate synthase ( $g l t A)$ (532 bp) (b), and heat-shock protein ( $\mathrm{rroEL}$ ) ( $815 \mathrm{bp)} \mathrm{genes} \mathrm{(c).} \mathrm{The} \mathrm{phylogenetic} \mathrm{trees} \mathrm{were} \mathrm{constructed} \mathrm{by} \mathrm{the} \mathrm{ML} \mathrm{method}$ (bootstrap replications: 1000) with Kimura 2-parameter plus Gamma rate model. The scale bar indicated the estimation of evolutionary distance. The taxa marked by bold font depict the sequences obtained in the study, and GenBank accession number is provided at the end of each strain name. ML, maximum likelihood. 


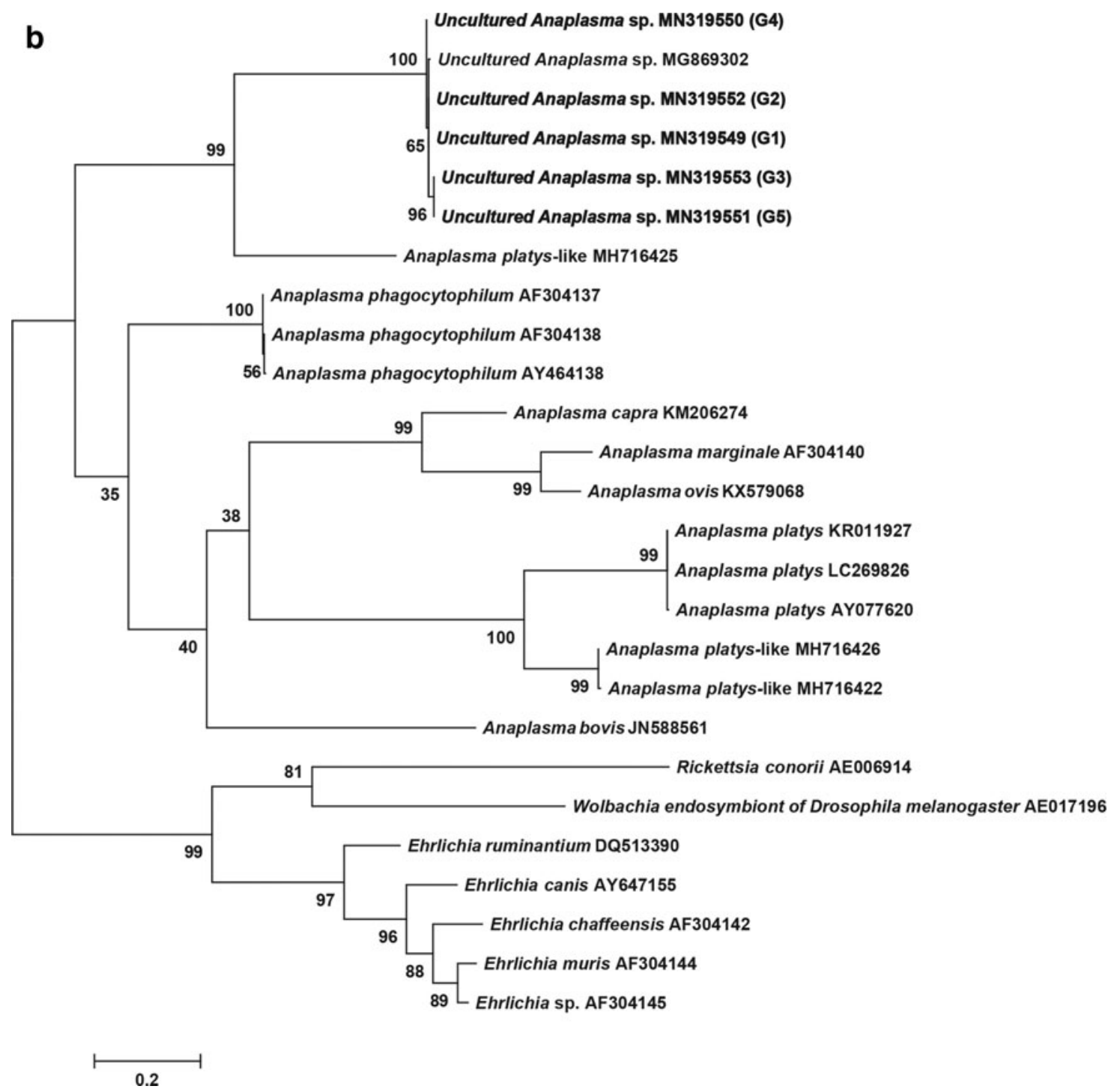

FIG. 2. (Continued).

(continued)

was genetically related to the A. platys and A. platys-like strains in Ankang (Fig. 2c) (Guo et al. 2018, 2019).

The couple of positive goats infected with Anaplasma sp. gave birth in our laboratory settings. We found that all the family members of the goats (G1-G5) were infected with this Anaplasma unexpectedly (Fig. 2), although there were some gene variations. The almost full-length $16 \mathrm{~S}$ rRNA sequences shared $100 \%$ nucleotide identities with each other and 99.93\% identities with the Anaplasma strain detected in the goats from Xi'an, China (Guo et al. 2018) (Fig. 2a). The sequences of the partial gltA gene showed 98.5-100\% similarity with each other and exhibited 99.07-99.87\% identities with Anaplasma sp. found in Xi'an (Fig. 2b). For the partial sequences of groEL gene, they showed 98.9-100\% nucleotide similarity with each other and presented 98.03-99.64\% identities with Anaplasma spp. identified in Xi'an (Fig. 2c). We only got the short-length gene sequence of $16 \mathrm{~S}$ rRNA of G1 and G2 but not the complete one; thus, we did not include them in the phylogenetic tree. We observed the offspring G3G5 with some symptoms of anaplasmosis, including inap- petence, high fever, vomiting, and weakness of limbs. During the examination, morulae were found in the platelet of the goats' family (G1-G5) in the blood smears by WrightGiemsa staining, but not in monocytes, neutrophils, and red blood cells (Fig. 3). Consequently, we named this bacterium A. platys-like.

\section{Discussion}

In this study, the goat couples $(\mathrm{G} 1, \mathrm{G} 2)$ were fed in a tickfree environment, and the goats G3-G5 were found to be all infected with $A$. platys-like bacterium. This suggests the vertical transmission of $A$. platys-like in goats. We also observed some clinical symptoms in the offspring, which might be caused by $A$. platys-like. By morphological examination of blood smears from the infected goats, we suggest that this Anaplasma might infect platelets.

In previous research, there are six species of the genus Anaplasma, which infect mammals and particular cell types of hosts such as erythrocytes, neutrophils, platelets, and 


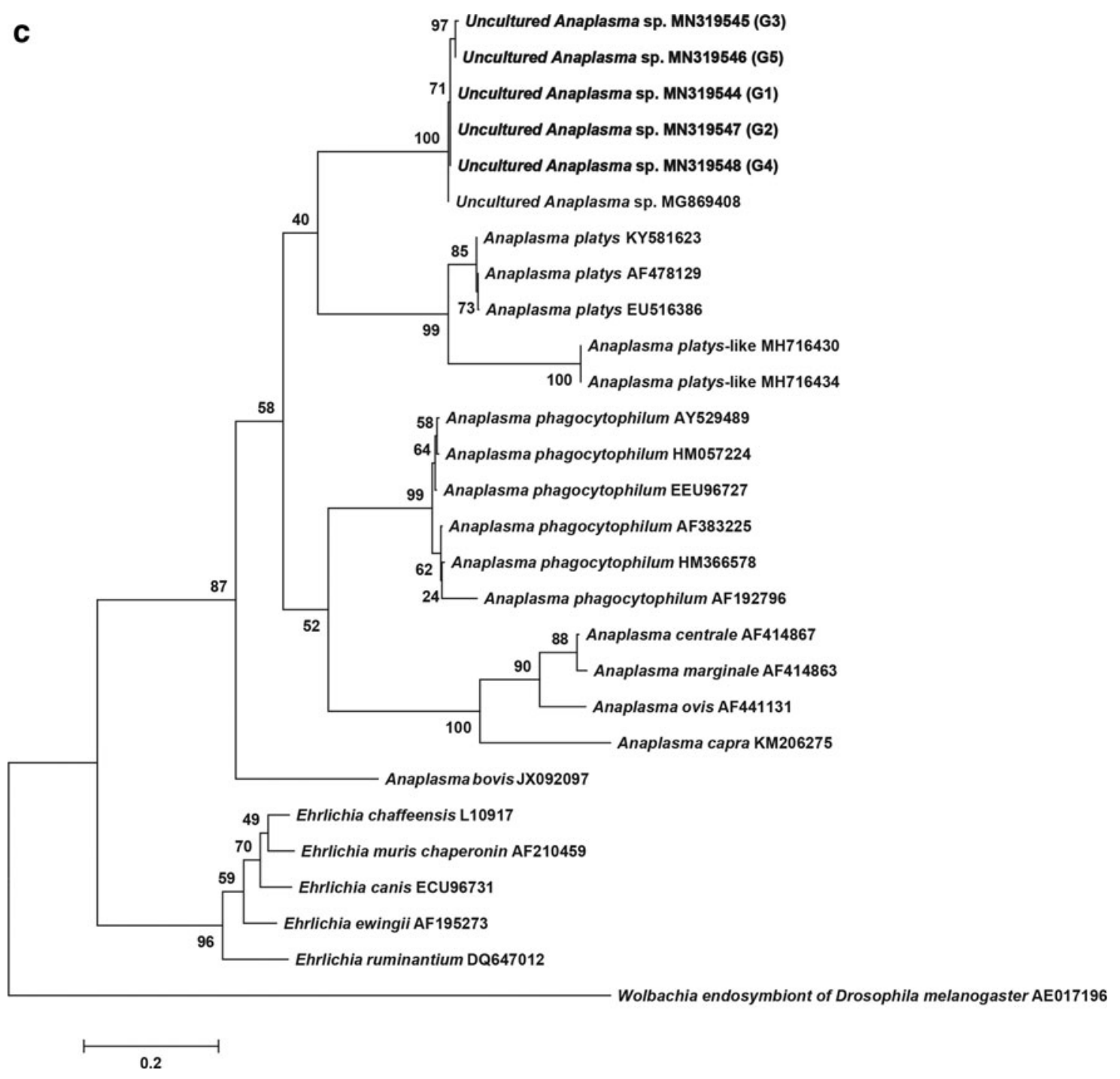

FIG. 2. (Continued).

monocytes (Dumler et al. 2001). Currently, A. platys is the only classified rickettsial species known to infect platelets, and caused cyclic thrombocytopenia in dogs (Dumler et al. 2001); this pathogen has also been found in cats (Lima et al. 2010), cattle, Camelus bactrianus, goats, Cervus elaphus, and African buffalo (Chochlakis et al. 2009, Dahmani et al. 2015, Li et al. 2015b, Lorusso et al. 2016a, 2016b, Machado et al. 2016). The investigation from Italy indicated that the prevalence of $A$. platys-like infection was higher in the goats than in the calves and sheep (Zobba et al. 2014), and it was also lower in the cattle in Algeria than in the calves in Italy (Dahmani et al. 2015). Through pathogen detection and symptom observation of the goat offspring (G3-G5), we suggest that there is a high probability that A. platys-like strain might impact goat husbandry, which deserves further epidemiological investigations and surveillance.

Up to date, there are two Anaplasma spp. that are recognized as human pathogens. For instance, A. phagocytophilum was the agent of human granulocytic anaplasmosis (Chen et al. 1994). A. capra was identified as a human pathogen in China recently (Li et al. 2015a). A. platys and A. ovis have been identified as the potential human pathogens (Chochlakis et al. 2010, Arraga-Alvarado et al. 2014). Whether A. platyslike identified in our study could infect human is an important issue to be clarified, however, we did not successfully isolate the bacterium. Further studies should be carried out to determine the pathogenicity of Anaplasma infected with platelets to humans.

To date, molecular and pathogenic identifications are still the significant methods of detecting Anaplasma, and serological testing is also commonly used as a supplementary detection. In our study, we identified the infection with Anaplasma by PCR and blood smear, but not by the antibody detection because A. platys-like strain isolate is not available. Based on the previous comparison on diagnosis methods, PCR was highly sensitive and endorsed for blood parasite diagnosis (Rucksaken et al. 2019, Wardrop et al. 2016). In addition, for PCR detection, it is worth noting that only targeting the $16 \mathrm{~S}$ rRNA gene is not sufficient for the identification of Anaplasma. Phylogenetic analysis based on the sequences of $16 \mathrm{~S}$ rRNA, gltA, and groEL indicated that A. platys-like in our study is similar to Anaplasma sp. identified 


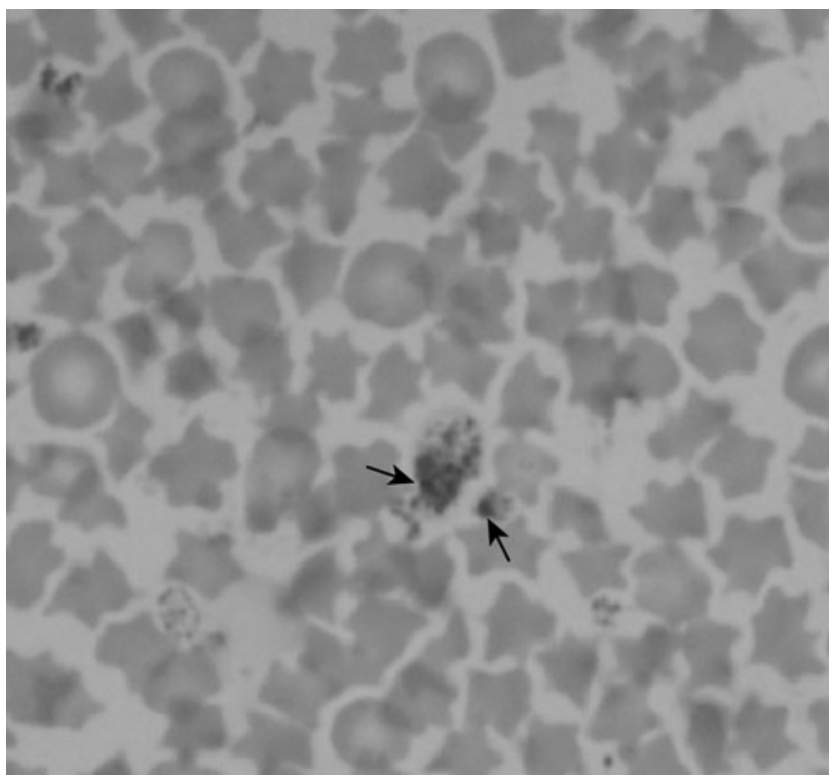

FIG. 3. The blood smear of Anaplasma platys-likeinfected goat was stained with the Wright-Giemsa staining method, and the inclusion is identified with arrowhead as blue-purple stained bodies within the platelet cytoplasm.

from goats in $\mathrm{Xi}$ 'an and is related to A. platys strains and A. platys-like in $R$. microplus in Ankang. With the phylogenetic analysis, the gltA and groEL sequences from the five goats (G1-G5) were not completely identical, which suggested that the G3-G5 might be coinfected with other Anaplasma sp. However, we performed molecular detection and blood smear observation, and found no other Anaplas$m a$ infection.

The previous reports also identified Anaplasma infection in domestic animals in northern China including Beijing, Inner Mongolia, Shanxi, and Hebei. The investigations in rural areas of Beijing showed that $48.9 \%$ (44/90) of goats, $23.9 \%(17 / 71)$ of cattle, and $0 \%(0 / 2)$ of dogs were infected with A. phagocytophilum by PCR amplification (Zhang et al. 2012). In Shanxi Province, $78.1 \%$ (25/32) of sheep were infected with Anaplasma spp., and 8.8\% (30/338) of goats were identified as A. capra positive (Li et al. 2012, Zhang et al. 2016, Peng et al. 2018). The study from Hebei indicated that A. capra was detected in 51.1\% (23/45) of sheep (Yang et al. 2017). The infection rate of $A$. capra in the goats in Inner Mongolia was $10 \%$ (4/40) (Peng et al. 2018). The seroprevalence of $A$. phagocytophilum was higher in rural residents from Tianjin (41.8\%) than from Beijing (13.6\%) (Zhang et al. 2014). According to the data described above, the infection rate of A. capra in the goats in our study was $44.6 \%$ (41/92), which was lower than in the sheep in Hebei (51.1\%). Until now, there has not an A. platys infection report in northern China.

Ticks play an important role in the transmission of Anaplasma (Rar et al. 2011). Rhipicephalus sanguineus is considered to be the primary vector of $A$. platys transmission (Simpson et al. 1991, Cicuttin et al. 2015, Li et al. 2015b). Rhipicephalus turanicus and Rhipicephalus microplus were reported to be infected with A. platys-like strains (Harrus et al. 2011, Lu et al. 2017), and A. capra (Guo et al. 2019).
The limitations of this study were as follows. Although we have observed that $A$. platys-like-infected goats had some symptoms, the veterinary significance of this bacterium should be further evaluated with a larger sample size. In addition, we found the possibility of vertical transmission, however, this observation was beyond our initial plan, and thus, no detailed procedure was designed to describe this transmission. Many critical characteristics were not available, such as what time was the offspring found positive after its birth. This should be evaluated in further animal models. Finally, the infection rate of A. platys-like in ticks is unknown, specially around the farm where we got goat samples.

\section{Conclusions}

In recent years, due to frequent contact between the rural and urban populations, the threat of TBDs to human health is not limited to rural areas. In our study, we detected A. platyslike in goats in Fangshan district, Beijing, which might infect platelets of goats and be a risk of vertical transmission although its pathogenicity to human was uncertain.

\section{Author Disclosure Statement}

No competing financial interests exist.

\section{Funding Information}

This work was supported by the Natural Science Foundation of China (81773492), State Key Research Development Program of China (2019YFC1200401, 2019YFC 1200202, 2019YFC1200501), Guangxi scientific and technological research (2020AB39264), and Special Program for Prevention and Control of Infectious Diseases in China (2018ZX10712001-018-001).

\section{References}

Arraga-Alvarado CM, Qurollo BA, Parra OC, Berrueta MA, et al. Molecular evidence of Anaplasma platys infection in two women from Venezuela. Am J Trop Med Hyg 2014; 91 : 1161-1165.

Botelho-Nevers E, Socolovschi C, Raoult D, Parola P. Treatment of Rickettsia spp. infections: A review. Expert Rev Anti Infect Ther 2012; 10:1425-1437.

Chen SM, Dumler JS, Bakken JS, Walker DH. Identification of a granulocytotropic Ehrlichia species as the etiologic agent of human disease. J Clin Microbiol 1994; 32:589595.

Chochlakis D, Ioannou I, Sharif L, Kokkini S, et al. Prevalence of Anaplasma sp. in goats and sheep in Cyprus. Vector Borne Zoonotic Dis 2009; 9:457-463.

Chochlakis D, Ioannou I, Tselentis Y, Psaroulaki A. Human anaplasmosis and Anaplasma ovis variant. Emerg Infect Dis 2010; 16:1031-1032.

Cicuttin GL, Tarragona EL, De Salvo MN, Mangold AJ, et al. Infection with Ehrlichia canis and Anaplasma platys (Rickettsiales: Anaplasmataceae) in two lineages of Rhipicephalus sanguineus sensu lato (Acari: Ixodidae) from Argentina. Ticks Tick Borne Dis 2015; 6:724-729.

Čobádiová A, Reiterová K, Derdáková M, Špilovská S, et al. Toxoplasma gondii, Neospora caninum and tick-transmitted 
bacterium Anaplasma phagocytophilum infections in one selected goat farm in Slovakia. Acta Parasitol 2013; 58:541546.

Dahmani M, Davoust B, Benterki MS, Fenollar F, et al. Development of a new PCR-based assay to detect Anaplasmataceae and the first report of Anaplasma phagocytophilum and Anaplasma platys in cattle from Algeria. Comp Immunol Microbiol Infect Dis 2015; 39:39-45.

Dumler JS, Barbet AF, Bekker CP, Dasch GA, et al. Reorganization of genera in the families Rickettsiaceae and Anaplasmataceae in the order Rickettsiales: Unification of some species of Ehrlichia with Anaplasma, Cowdria with Ehrlichia and Ehrlichia with Neorickettsia, descriptions of six new species combinations and designation of Ehrlichia equi and 'HGE agent' as subjective synonyms of Ehrlichia phagocytophila. Int J Syst Evol Microbiol 2001; 51:21452165.

Garcia-Perez AL, Barandika J, Oporto B, Povedano I, et al. Anaplasma phagocytophila as an Abortifacient Agent in Sheep Farms from Northern Spain. Ann N Y Acad Sci 2003; 990:429-432.

Geurden T, Somers R, Thanh NT, Vien LV, et al. Parasitic infections in dairy cattle around Hanoi, Northern Vietnam. Vet Parasitol 2008; 153:384-388.

Guo WP, Huang B, Zhao Q, Xu G, et al. Human-pathogenic Anaplasma spp., and Rickettsia spp. in animals in Xi'an, China. PLoS Negl Trop Dis 2018; 12:e0006916.

Guo WP, Zhang B, Wang YH, Xu G, et al. Molecular identification and Characteriza-tion of Anaplasma capra and Anaplasma platys-like in Rhipicephalus microplus in Ankang, Northwest China. BMC Infect Dis 2019; 19:434.

Harrus S, Perlman-Avrahami A, Mumcuoglu KY, Morick D, et al. Molecular detection of Ehrlichia canis, Anaplasma bovis, Anaplasma platys, Candidatus Midichloria mitochondrii and Babesia canis vogeli in ticks from Israel. Clin Microbiol Infect 2011; 17:459-463.

Hendrix GK, Brayton KA, Burcham GN. Anaplasma ovis as the suspected cause of mortality in a neonatal elk calf. J Vet Diagn Invest 2019; 31:267-270.

Ismail N, Bloch KC, McBride JW. Human ehrlichiosis and anaplasmosis. Clin Lab Med 2010; 30:261-292.

Li GH, Yu Q, Zhang QX, Pan L, et al. [Molecular epidemiology regarding Anaplasma phagocytophilum in Dermacentor silvarum in Ningwu county, Shanxi province]. Zhonghua Liu Xing Bing Xue Za Zhi 2012; 33:642-643.

Li H, Zheng YC, Ma L, Jia N, et al. Human infection with a novel tick-borne Anaplasma species in China: A surveillance study. Lancet Infect Dis 2015a; 15:663-670.

Li Y, Yang J, Chen Z, Qin G, et al. Anaplasma infection of Bactrian camels (Camelus bactrianus) and ticks in Xinjiang, China. Parasit Vectors 2015b; 8:313.

Lima ML, Soares PT, Ramos CA, Araújo FR, et al. Molecular detection of Anaplasma platys in a naturally-infected cat in Brazil. Braz J Microbiol 2010; 41:381-385.

Liu Z, Ma M, Wang Z, Wang J, et al. Molecular survey and genetic identification of Anaplasma species in goats from central and southern China. Appl Environ Microbiol 2012; 78:464-470.

Lorusso V, Wijnveld M, Latrofa MS, Fajinmi A, et al. Canine and ovine tick-borne pathogens in camels, Nigeria. Vet Parasitol 2016a; 228:90-92.

Lorusso V, Wijnveld M, Majekodunmi AO, Dongkum C, et al. Tick-borne pathogens of zoonotic and veterinary importance in Nigerian cattle. Parasit Vectors 2016b; 9:217.
Lu M, Tian JH, Yu B, Guo WP, et al. Extensive diversity of rickettsiales bacteria in ticks from Wuhan, China. Ticks Tick Borne Dis 2017; 8:574-580.

Machado RZ, Teixeira MM, Rodrigues AC, André MR, et al. Molecular diagnosis and genetic diversity of tick-borne Anaplasmataceae agents infecting the African buffalo Syncerus caffer from Marromeu Reserve in Mozambique. Parasit Vectors 2016; 9:454.

Mathieson AO. Ectoparasites and their control. In: Martin WB, Aitken ID, eds. Diseases of Sheep, 2nd ed. Oxford: Blackwell Science Publication, 1991:284-289.

Peng Y, Wang K, Zhao S, Yan Y, et al. Detection and phylogenetic characterization of: An emerging pathogen in sheep and goats in China. Front Cell Infect Microbiol 2018; 8:283.

Perronne C. Lyme and associated tick-borne diseases: Global challenges in the context of a public health threat. Front Cell Infect Microbiol 2014; 4:74.

Rar V, Golovljova I. Anaplasma, Ehrlichia, and "Candidatus Neoehrlichia" bacteria: Pathogenicity, biodiversity, and molecular genetic characteristics, a review. Infect Genet Evol 2011; 11 : 1842-1861.

Reppert E, Galindo RC, Breshears MA, Kocan KM, et al. Demonstration of transplacental transmission of a human isolate of Anaplasma phagocytophilum in an experimentally infected sheep. Transbound Emerg Dis 2013; 60 (Suppl.): 93-96.

Rucksaken R, Maneeruttanarungroj C, Maswanna T, Sussadee $\mathrm{M}$, et al. Comparison of conventional polymerase chain reaction and routine blood smear for the detection of Babesia canis, Hepatozoon canis, Ehrlichia canis, and Anaplasma platys in Buriram Province, Thailand. Vet World 2019; 12: 700-705.

Silaghi C, Santos AS, Gomes J, Christova I, et al. Guidelines for the direct detection of Anaplasma spp. in diagnosis and epidemiological studies. Vector Borne Zoonotic Dis 2017; 17: 12-22.

Simpson RM, Gaunt SD, Hair JA, Kocan KM, et al. Evaluation of Rhipicephalus sanguineus as a potential biologic vector of Ehrlichia platys. Am J Vet Res 1991; 52:1537-1541.

Stuen S. Anaplasma phagocytophilum-The most widespread tick-borne infection in animals in Europe. Vet Res Commun 2007; 31(Suppl.):79-84.

Stuen S, Granquist EG, Silaghi C. Anaplasma phagocytophilum a widespread multi-host pathogen with highly adaptive strategies. Front Cell Infect Microbiol 2013; 3:31.

Tamura K, Peterson D, Peterson N, Stecher G, et al. MEGA5: Molecular evolutionary genetics analysis using maximum likelihood, evolutionary distance, and maximum parsimony methods. Mol Biol Evol 2011; 28:2731-2739.

Tate CM, Howerth EW, Mead DG, Dugan VG, et al. Anaplasma odocoilei sp. Nov.(family Anaplasmataceae) from white-tailed deer (Odocoileus virginianus). Ticks Tick Borne Dis 2013; 4:110-119.

Tay ST, Koh FX, Kho KL, Ong BL. Molecular survey and sequence analysis of Anaplasma spp. in cattle and ticks in a Malaysian farm. Trop Biomed 2014; 31:769-776.

Torina A, Vicente J, Alongi A, Scimeca S, et al. Observed prevalence of tick-borne pathogens in domestic animals in Sicily, Italy during 2003-2005. Zoonoses Public Health 2007; 54:8-15.

Wardrop KJ, Birkenheuer A, Blais MC, Callan MB, et al. Update on canine and feline blood donor screening for bloodborne pathogens. J Vet Intern Med 2016; 30:15-35. 
Yang J, Han R, Niu Q, Liu Z, et al. Occurrence of four Anaplasma species with veterinary and public health significance in sheep, northwestern China. Ticks Tick Borne Dis 2018; 9: 82-85.

Yang J, Liu Z, Niu Q, Liu J, et al. A novel zoonotic Anaplasma species is prevalent in small ruminants: Potential public health implications. Parasit Vectors 2017; 10:264.

Yasini S, Khaki Z, Rahbari S, Kazemi B, et al. Hematologic and clinical aspects of experimental ovine anaplasmosis caused by Anaplasma ovis in Iran. Iran J Parasitol 2012; 7: 91-98.

Zhang L, Liu H, Xu B, Zhang Z, et al. Rural residents in China are at increased risk of exposure to tick-borne pathogens Anaplasma phagocytophilum and Ehrlichia chaffeensis. Biomed Res Int 2014; 2014:313867.

Zhang XC, Zhang LX, Li WH, Wang SW, et al. Ehrlichiosis and zoonotic anaplasmo-sis in suburbanareas of Beijing, China. Vector Borne Zoonotic Dis 2012; 12:932937.

Zhang Y, Lv Y, Zhang F, Zhang W, et al. Molecular and phylogenetic analysis of Anaplasma spp. in sheep and goats from six provinces of China. J Vet Sci 2016; 17:523529.

Zobba R, Anfossi AG, Pinna Parpaglia ML, Dore GM, et al. Molecular investigation and phylogeny of Anaplasma spp. in Mediterranean ruminants reveal the presence of neutrophiltropic strains closely related to A. platys. Appl Environ Microbiol 2014; 80:271-280.
Address correspondence to: Yan-Ling $\mathrm{Hu}$

Life Sciences Institute

Guangxi Medical University

22 Shuangyong Street

Nanning, Guangxi 530021

China

E-mail: huyanling@gxmu.edu.cn

Na Jia

State Key Laboratory of Pathogen and Biosecurity Beijing Institute of Microbiology and Epidemiology 20 Dong-Da Street Fengtai, Beijing 100071

China

E-mail: jiana7941@ @otmail.com

Wu-Chun Cao

State Key Laboratory of Pathogen and Biosecurity Beijing Institute of Microbiology and Epidemiology 20 Dong-Da Street Fengtai, Beijing 100071

China

E-mail: caowc@bmi.ac.cn 